\title{
La integración social: Un desafío en la política de desarrollo local sostenible en la parroquia urbana la providencia del cantón Machala
}

Social integration: A challenge in the policy of sustainable local development in the urban parish la providencia of Machala city

Alba Violeta Granda Granda. ${ }^{1}$, Viviana Verónica Carriel Bustamante. ${ }^{2}$ \& Yonimiler Castillo Ortega. ${ }^{3}$

Recibido: 14-11-2020 / Revisado: 28-11-2020 /Aceptado: 17-12-2020/ Publicado: 02-01-2021

\begin{abstract}
.
DOI: https://doi.org/10.33262/cienciadigital.v5i1.1528

The purpose of this article is to establish the policies that allow achieving sustainable local development oriented towards social integration in the urban parish La Providencia of Machala city. For this purpose, leaders of 22 marginal urban neighborhoods of the parish participated, as well as departmental heads of the cantonal decentralized autonomous government (DAG) of Machala. Among the research techniques that were applied were the questionnaire, the interview and the observation, the same ones that were applied through structured instruments (interview guide, survey form and observation guide) as well as bibliographic techniques for the theoretical foundation of the object study. The study shows that despite the existence of public policies oriented through regulations such as: Organic Code of Territorial Organization, Autonomies and Decentralization (OCTOAD), National Development Plan (NDP), Land Management Plan (LMP), legal instruments and technicians aimed at achieving the well-being of its inhabitants, the latter, elaborated by the cantonal DAG of Machala, these norms are implemented more to comply with the requirements of the control organisms, than to achieve the true wellbeing of their territory. In this sense, planning obeys the political vision of the current
\end{abstract}

\footnotetext{
1 Universidad Católica de Cuenca, Posgrado, Maestría en Desarrollo Local Mención en Ordenamiento Territorial, Cuenca Ecuador, alba.granda@est.ucacue.edu.ec, https://orcid.org/0000-0002-0021-5023

${ }^{2}$ Universidad Católica de Cuenca Carrera de economía, Cuenca, Ecuador, viviana.carriel@ucacue.edu.ec, https://orcid.org/0000-0001-6572-0648

3 Universidad Católica de Cuenca, Posgrado, Maestría en Desarrollo Local Mención en Ordenamiento Territorial, Cuenca Ecuador, ycastilloo@ucacue.edu.ec, https://orcid.org/0000-0002-7710-5199
} 
ruler, in a kind of political client neglecting the true needs of the inhabitants of the sector under study, a situation that is evidenced in the lack of basic services and community infrastructure as described in this article. Based on these findings, it remains a challenge to achieve a social integration that guarantees access of all neighborhoods to basic services to improve the quality of life of the inhabitants of Machala city, particularly in La Providencia parish.

Keywords: Social integration, sustainable local development, development policies

\section{Resumen.}

El presente artículo tiene como propósito establecer las políticas que permitan lograr el desarrollo local sostenible orientado a la integración social en la parroquia urbana La Providencia del cantón Machala. Para cuyo efecto participaron dirigentes de 22 barrios urbano marginales de la parroquia, así como también jefes departamentales del GAD cantonal de Machala entre las técnicas de investigación que se aplicaron fueron el cuestionario, la entrevista y la observación, mismas que se aplicaron mediante instrumentos estructurados (guía de entrevista, boleta de encuesta y guía de observación) así como también técnicas bibliográficas para la fundamentación teórica del objeto de estudio. En el estudio se demuestra que a pesar de la existencia de políticas públicas orientadas a través de normativas tales como: Código Orgánico de Organización Territorial, Autonomías y Descentralización (COOTAD), Plan Nacional de Desarrollo, Plan de Ordenamiento Territorial (PDyOT), instrumentos legales y técnicos encaminadas a lograr el bienestar de sus habitantes, este último, elaborado por el GAD cantonal de Machala, dichas normas se implementan más por cumplir con las exigencias de los organismos de control, que por lograr el verdadero bienestar de su territorio. En ese sentido la planificación obedece a la visión política del gobernante de turno, en una especie de clientelismo político descuidando las verdaderas necesidades de los habitantes del sector objeto de estudio, situación que se evidencia en la carencia de servicios básicos e infraestructura comunitaria como se describe en el presente artículo, consecuentemente continua siendo un desafío para la ciudad de Machala particularmente en la parroquia La providencia lograr una integración social que garantice el acceso a todos los barrios a servicios necesarios para mejorar la calidad de vida de sus habitantes.

Palabras claves: Integración social, desarrollo local sostenible, Políticas de desarrollo.

\section{Introducción.}

América Latina y el Caribe enfrentan tres grandes desafíos: sociales, económicos y políticos estos son reducir la pobreza, aumentar su competitividad y consolidar su integración, consecuentemente necesita con urgencia un fuerte impulso y un reordenamiento de sus prioridades, dando un mayor énfasis al combate contra a la pobreza. En ese sentido, se han 
planteado políticas de Desarrollo Social que son un conjunto de medidas, con el fin de crear y promover las condiciones sociales, económicas, políticas, culturales y jurídicas que faciliten el acceso a la población total a beneficios de desarrollo de manera igualitaria y equitativa, para cuyo efecto el Programa de las Naciones Unidas para el Desarrollo (PNUD), tiene como función contribuir a mejorar la calidad de vida de las naciones y promover el cambio, para ayudar a los pueblos a forjar una vida mejor.

Para el caso de Ecuador, la situación no es distinta, pues también afronta retos en temas políticos, económicos y sobre todo sociales, y estos se dimensionan con más fuerza por las desigualdades existentes, ante esto es necesario establecer y puntualizar las características de estas problemáticas, con el fin de buscar un tratamiento e implementar estrategias encaminadas a lograr el desarrollo local sostenible como proceso integral de transformaciones sociales, económicas y ambientales.

Lo anteriormente señalado es posible con la participación de los actores sociales identificando sus necesidades básicas, para planificar, ejecutar y tomar decisiones aplicando estrategias y objetivos que garanticen su desarrollo, mejorar el nivel y la calidad de vida, así como también, enriquecer los valores de la población y reforzar las potencialidades existentes en las diferentes localidades, tanto en recursos naturales como humanos y culturales para ser aprovechados como elementos dinamizadores de un desarrollo desde lo interno.

En ese sentido, las demandas sociales identificadas servirán de insumo para establecer si las políticas públicas planteadas están siendo orientadas a mejorar la calidad de vida de sus habitantes y garantizar los derechos constitucionales, como referente obligado para que las instituciones del Estado en los diversos niveles de gobierno, mejoren, amplíen y doten de calidad sus intervenciones para responder a los problemas públicos en el corto, mediano y largo plazo a fin de promover el desarrollo integral de la sociedad.

Al respecto, la constitución del Ecuador en uno de sus artículos manifiesta el ejercicio de los derechos y señala que: Todas las personas son iguales y gozarán de los mismos derechos, deberes y oportunidades, así como también, menciona que el Estado adoptará medidas de acción afirmativa que promuevan la igualdad real en favor de los titulares de derechos que se encuentren en situación de desigualdad, en ese sentido, el estado ecuatoriano garantiza que las personas, comunidades y pueblos gozarán de los derechos amparados en la Constitución 2008 y en los instrumentos internacionales estos se podrán ejercer y exigir ante autoridades competentes para su cumplimiento.

A esto se incorpora los derechos del buen vivir como un principio constitucional basado en el Sumak Kawsay, que recoge una visión del mundo centrada en el ser humano, como parte de un entorno natural y social; derecho al agua y alimentación, ambiente sano, comunicación e información, cultura y ciencia, educación, vivienda, salud, trabajo y seguridad social. 
Por su parte, el Gobierno Nacional ha establecido la necesidad de incorporar un enfoque territorial en visión del desarrollo, con el propósito de dar integridad y sostenibilidad a los procesos relacionados con la planificación y el ordenamiento del territorio ecuatoriano; en ese sentido, el Gobierno Autónomo Descentralizado Provincial de El Oro (GADPEO) asume como prioridad y centro de su accionar al ser humano, sus necesidades y aspiraciones; $\mathrm{y}$ conforme a los marcos legales vigentes en el país, ha asumido la responsabilidad de reformular el Plan de Desarrollo y Ordenamiento Territorial de la Provincia de El Oro 20142025, considerándolo como una herramienta fundamental para responder a las demandas y aspiraciones de desarrollo de todos los Orenses y generar políticas que permitan el desarrollo local sostenible encaminadas a lograr una verdadera integración social

En tal virtud, en el presente artículo se describen las manifestaciones de estas desigualdades y que ameritan su intervención en procura de lograr la ansiada integración social, como es el caso de La Providencia que es una parroquia urbana que pertenece al Cantón Machala, está ubicada al noreste de la ciudad y está compuesta por 84 barrios, de los cuales 22 se encuentran excluidos de la planificación urbana, que conlleva a una marginación y exclusión social lo que implica carecer de necesidades vitales obligatorias es decir que no poseen los servicios básicos más elementales, consecuentemente no gozan de una vida digna, aunque en la Planificación del Desarrollo y el Ordenamiento Territorial (actualización 2018) del cantón Machala tiene como finalidad proteger los recursos naturales del territorio y propender a mejorar las condiciones de vida de sus habitantes, sin embargo la planificación y desarrollo urbanístico de vastos sectores populares no llega.

Por esta razón es necesario contar con un plan de desarrollo que regule y proponga estrategias generales y específicas de manera integrada orientada a dar respuesta a los problemas socio territoriales derivados de la falta de integración económica y social que se traduce en la alteración ecológica y ambiental, la concentración espacial de la población en áreas urbanas, la degradación de espacios naturales de especial valor ecológico y la tendencia de un modelo de consumo de recursos naturales de manera indiscriminada.

Lo anteriormente descrito se debe a que el proceso de planificación en nuestra provincia y particularmente en el Cantón Machala, no está desarrollado integralmente, existiendo falencias en términos de articulación y coordinación entre niveles de gobierno, lo que no permite establecer estrategias que procuren resolver problemas comunes, basados en los principios de participación y concertación institucionales y la utilización de recursos que generen sinergias para la acumulación de rendimientos y el logro de los objetivos planteados.

En tal virtud, es importante la aplicación de las políticas públicas existentes en la planificación del GAD Machala, en lo relacionado a gestión, planificación y monitoreo de la percepción, intereses y requerimientos de la sociedad que permita trabajar en un permanente encuentro a lo interno y externo, con los gobiernos locales, para el desarrollo de nuestro entorno. 


\section{Aspectos teóricos sobre el desarrollo local sostenible}

La palabra desarrollo aparece en la agenda social después de la segunda Guerra mundial, en la recomposición del Orden mundial que inicia con la división en dos mundos: el occidental o capitalista y por otro lado el oriental o socialista, con el fin de reconstruir la sociedad golpeada por la Guerra. En este llamado nuevo orden mundial, gran parte de la humanidad queda excluida del sistema mundial, a la que denominaron países del tercer mundo, en donde, no se empleó la palabra reconstrucción, sino que se utilizó la palabra desarrollo, es decir este grupo humano debía crecer para desenvolverse con relativa autonomía. De ese modo, después de la segunda Guerra mundial el mundo debía reconstruirse por una parte y desarrollarse por otra. (Arocena, 2002)

En ese sentido "el desarrollo se presenta como un posicionamiento diferenciado y asimétrico de los individuos y de los países en los procesos de producción y de distribución que se acompaña de disparidades y desequilibrios territoriales y sectoriales". (Mendieta, 2009,p.2)

No obstante, se han presentado diversas corrientes sobre el desarrollo; así, las ideas neoclásicas de progreso asumen el aumento de productividad del trabajo, y el consecuente aumento de los salarios, como resultado de la acumulación de capital, teoría que fue cuestionada tras la sobreproducción alcanzada por los países hegemónicos, la cual los llevo a implementar un imperialismo que desató la primera guerra mundial. Por otro lado, tenemos a Shumpeter que plantea una diferencia frente a los neoclásicos e incluye la importancia de la transformación de los procesos productivos mediante las innovaciones y avances tecnológicos, sugiriendo que las innovaciones son necesarias para el desarrollo.

Los postulados neoclásicos son relevados por Keynes, quién reconoce la persistencia de desequilibrios, como el desempleo y que es necesario la intervención del Estado que defina a la política económica como la mejor herramienta para salir de una crisis, mediante la regulación de los mercados y la adopción de políticas que estimulen el crecimiento mediante el gasto público y así aumentar la producción, la inversión y el empleo.

Finalmente, la Teoría Económica institucional de Douglas Nouth que menciona que las instituciones determinan que el país crezca o no, las instituciones son las reglas de juego formal e informal que determinan, orientan, incentivan las acciones de las personas de cada sociedad. Las reglas formales serían las leyes, la constitución, el congreso, el Estado, como principal generador de instituciones, entregando incentivos a la inversión, al trabajo y al ahorro; las reglas infórmales vendrían a ser los valores, los hábitos, que determina las acciones de la sociedad (Mendieta, 2009).

Los autores Mujica \& Rincón, manifiestan que "el desarrollo es concebido como un proceso de cambio social, deliberado, cuyo objetivo último es la igualación de oportunidades sociales, políticas y económicas, tanto en el plano nacional como en las relaciones con otras sociedades más avanzadas" (2010,p.6). Por su parte Sen. (1998) sostiene que toda política de desarrollo 
debe garantizar el bienestar, la igualdad, la mejora de la calidad de vida y resguardar los derechos humanos como impulso del desarrollo en beneficio de todos los habitantes. Esto permite también referirse a diferentes aspectos que se desintegran del desarrollo como: lo local, lo humano, y lo sostenible.

Lo antes descrito permite analizar y comprender el desarrollo local en la visión de los autores Alburquerque (2004) y González-Lendizabal et al., (2016), quienes señalan que el desarrollo local aparece en la década de los setenta del siglo XX en la crisis y caída de los modelos de crecimiento tradicionales que sufrieron los países desarrollados de Europa Occidental. Este suceso dio paso a pensar en otra forma de progreso que superara al primero, provocando el cambio del modo de desarrollo a "lo pequeño y lo local", orientado a promover la participación social individual y colectiva a través de iniciativas locales en diversas áreas.

En esa misma dirección Gallicchio describe al desarrollo local como una "estrategia sociopolítica de cambio" (2010,p.13) coinciden también Irausquín et al., (2017) quienes plantean que el desarrollo local resalta la participación de los actores locales por mejorar la calidad de vida en sus localidades, en respuesta a las fallas de los mercados y de las políticas de los gobiernos nacionales para proveer lo que se requiere en los territorios.

Por otro lado los autores Aguado Moralejo et al., (2009) al igual que Irausquín et al., (2017) manifiestan que el concepto desarrollo sostenible surge en la década de los setenta a medida que el enfoque económico de desarrollo denotara sus fallas, las cuales comienzan a generar contradicciones ante su crecimiento sin límites y consumo desmesurado de los recursos naturales del planeta. Ante ello surge la necesidad de crear estrategias globales que cubrieran aspectos económicos, sociales y ambientales, en ese contexto surge la idea del "Desarrollo Sostenible" que además de buscar garantizar el equilibrio entre el crecimiento económico, el cuidado del medio ambiente haciendo uso los recursos naturales de forma racional, se interesa por generar igualdad, satisfacer las necesidades de las presentes y futuras generaciones.

Por su parte García Batiz et al. (2016) menciona que en el reporte llamado Nuestro futuro común de la World Commision on Environment and Development (WCED), de 1987, se define al termino desarrollo sostenible como aquel desarrollo que satisface las necesidades de las presentes generaciones sin comprometer las necesidades de las generaciones futuras. Del mismo modo la Asamblea General de las Naciones Unidas (2012) y el WBCSD ${ }^{4}$ (1999) citado por Boada Ortiz et al. (2005), definen el desarrollo sostenible como un sistema de producción y consumo capaz de asegurar una mejor equidad, calidad de vida y bienestar ambiental para las generaciones de hoy y del futuro, en tal virtud, el desarrollo sostenible fue determinado a partir de una visión global y de respeto hacia el futuro de nuestro planeta.

\footnotetext{
${ }^{4}$ Consejo Empresarial Mundial para el Desarrollo Sostenible, también conocido por sus siglas en inglés WBCSD
} 
Por su parte los autores Mora Mayoral \& Martínez Martínez hacen referencia a que:

"el termino desarrollo sostenible integra una serie de sostenibilidades conocidas como el triángulo de la sostenibilidad, que incluye lo ambiental, lo económico y lo social y explica que para asegurar que estos tres tipos de sostenibilidad se cumplan, es necesaria la existencia de un marco político-institucional que asegure los principios básicos para lograr un desarrollo sostenible" (2018,p.33)

Frente a esto Rodrigo \& Arenas. (2014) señalan que, una de las claves para lograr el desarrollo sostenible (DS) es el establecimiento de alianzas y mecanismos de colaboración entre diversos actores públicos, privados y de la sociedad civil a nivel mundial, regional, nacional y local, ya que con acciones separadas donde cada uno establece su propia agenda, difícilmente se podrá llegar a soluciones para los desafíos que demanda el DS.

Por lo mencionado se puede afirmar que, el termino desarrollo sostenible surge como una estrategia que a más de los tres aspectos (ambiental, económico y social) se debe incorporar el aspecto político para la toma de decisiones, considerando que este tipo de desarrollo debe adecuarse a contextos internacionales, nacionales y locales basándose en principios como equidad entre generaciones, equidad de género, reducción de la pobreza, preservación y restauración del medio ambiente, conservación de los recursos naturales y justicia social para lograr de manera equilibrada, el desarrollo económico, el desarrollo social y la protección del medio ambiente.

$\mathrm{Al}$ respecto Morales Pérez menciona que:

La Conferencia de las Naciones Unidas sobre Medio Ambiente y Desarrollo, en junio de 1992, no habla de un desarrollo económico local, sino de desarrollo local sostenible, como aquel promovido y desarrollado por autoridades locales en pro del desarrollo sostenible de su comunidad, para actuar hacia la mejora ambiental del municipio y como un proceso donde la forma local de gobierno, ampliamente comunitaria y participativa, tiene por objetivo establecer una estrategia de acción para la protección del medio ambiente, la prosperidad y el bienestar social dentro del ámbito local. (2006,p.64)

En tal virtud, en el plano territorial es importante diseñar instrumentos y políticas públicas de gestión dirigidas a estimular el aprovechamiento de los recursos locales para impulsar nuevos estilos de desarrollo basados en las potencialidades de las economías locales. Y que ese desarrollo posibilite la generación de servicios e infraestructuras que permita mejorar la calidad de vida de los habitantes, eso implica además garantizar el bienestar y satisfacción de las necesidades humanas fundamentales para lograr integración social y superar la exclusión social o pobreza y promover el desarrollo social de toda la población. 


\section{Políticas públicas e integración social}

Para abordar el tema de integración social es necesario conceptualizar lo opuesto, esto es, Segregación Social, así mismo abordar qué políticas públicas es necesario aplicar para revertir esta problemática social, a continuación, se detalla algunas concepciones que hacen varios autores sobre el tema:

Sánchez Steiner (2008) y Madrid et al. (2019), coinciden al afirmar que en el siglo XX en Latinoamérica se dieron procesos migratorios internos desde el campo a la ciudad es decir que la población campesina se apropió del espacio urbano donde estableció su vivienda, esto genero procesos acelerados de urbanización. Como lo indica Godoy (2019), esto acarreo un enorme problema a las grandes ciudades, es decir, se generó la segregación territorial. A este fenómeno Rodríguez \& Arriagada lo conciben como "formas de desigual distribución de grupos de población en el territorio, que se manifiestan de diferentes maneras" (2004,p.6)

Esta división de grupos no solo ha implicado la concentración de pobreza si no que, además, ha conllevado una diferencia objetiva entre ellos, lo que se reflejada en la desigualdad de equipamientos comunitarios y servicios de calidad, visible al compararse con los distintos sectores de la ciudad (Godoy, 2019). Consecuentemente es necesario identificar las posibilidades de romper con la segregación y lograr la integración territorial, económica y social.

Larraín \& Razmilic (2019) conceptualizan a la integración social desde dos dimensiones, la primera corresponde a la equidad, es decir, al igual acceso a bienes públicos y la segunda a la diversidad social o sea la proximidad entre grupos sociales, entendiendo la integración social como el acceso a oportunidades de trabajo, salud, educación, infraestructura y servicios.

La Unión Europea en El Libro Verde sobre la cohesión territorial (Europea, 2008) señala que la integración social también podría definirse como un principio para la actuación de políticas públicas encaminadas a favorecer el acceso equitativo a servicios, equipamientos y al desarrollo territorial para romper las actuales tendencias de polarización y desigualdad entre territorios para lograr este propósito deben conjugarse tres elementos esenciales:

La articulación física entre las partes del territorio a través de redes de infraestructura y la accesibilidad a servicios públicos como se señala anteriormente.

La equidad territorial, entendida como la igualdad de oportunidades para alcanzar el desarrollo de la persona en todas las partes de un territorio mediante la prestación de servicios públicos, equipamientos e infraestructuras en todo el territorio.

La identificación de la comunidad que habita en un territorio es más eficaz con un proyecto de vida en común, toda vez que éste los une, generando un sentido de pertenencia e identidad 
que permite afianzar los dos elementos antes descritos y minimiza cualquier aspecto que amenace la integración social.

En consecuencia, con lo afirmado en líneas anteriores, se entiende que la integración social es el resultado de la reducción de sistemas marginales que posibiliten a las personas que se encuentran en estado de segregación, ser parte del nivel de bienestar social, ya que esto potencia la autoestima personal y eleva el bienestar individual y colectivo.

Lograr la integración social es un problema bastante complejo en sociedades capitalistas como la nuestra, toda vez que revertir la tendencia de exclusión y segregación, para posibilitar que personas en riesgo de pobreza o de exclusión tengan la oportunidad de participar de manera plena en la vida social y puedan disfrutar de un nivel de vida adecuado es complicado por la casi nula implementación de políticas sociales de los gobiernos nacionales y locales.

Por su lado Abad (2013) y Velásquez (2009) coinciden en señalar que las políticas públicas son acciones gubernamentales como respuesta respecto a una o varias demandas de la sociedad. De este modo se puede decir que las Políticas públicas son un conjunto de decisiones emitidas por el gobierno para dar solución a un problema público. Por su parte Jiménez \& Espejel (2019) mencionan que, las políticas públicas deberán crear condiciones de confianza y transparencia con enfoque integral para de ese modo incentivar comportamientos comunitarios, inclusivos y así conformar una economía local sustentable.

En ese sentido, la constitución del Estado ecuatoriano incluye instrumentos internacionales y actúa en respeto y reconocimiento de los derechos de sus ciudadanos, esto implica reconocer los niveles mínimos de satisfacción de las necesidades humanas básicas para la sociedad, asumir y garantizar para aquellos grupos sociales que se encuentran excluidos, la satisfacción de estas necesidades como vía de integración social y acceder a lograr el buen vivir como un derecho fundamental del ser humano.

Al respecto la Secretaria Tecnica Planifica Ecuador en el informe de Avance del cumplimiento de la Agenda 2030 para el Desarrollo Sostenible señala que:

El nivel nacional propone la implementación de los Objetivos de Desarrollo Sostenible (ODS), su aplicación concreta se realiza a través de la gestión territorial de los Gobiernos Autónomos Descentralizados y de las entidades desconcentradas en el territorio, entendido como la expresión de la acción colectiva, mediante una planificación territorial actualizada y fortalecida como insumo de partida. (2019,p.20)

Lo anteriormente anotado supone que se cuenta con el andamiaje jurídico que respalda las políticas sociales encaminadas a lograr una verdadera integración; sin embargo, en el mundo real de las políticas, encontramos algunas que se alejan de lo público o del interés común, 
porque grupos particulares políticamente dominantes permean las esferas de poder para obtener a través de la política una ventaja en desmedro de los intereses de una colectividad.

\section{Integración Social en Machala, Ecuador}

La situación en Ecuador no es distinta, Carpio et al. (1987) al igual que Albornoz, (2011) mencionan que, como resultado de la modernización capitalista que se aceleró con la producción y exportación petrolera, se consolidaron tendencias de concentración de la economía. Estos procesos de urbanización, concentradores y excluyentes ciudades primadas ${ }^{5}$ generaron desigualdades de crecimientos urbanos a nivel nacional, frente al resto de ciudades y pueblos pequeños del país.

En el caso de la ciudad de Machala, la ampliación del territorio urbano se dio en torno a la producción primaria agroexportadora generadora de mano de obra y consecuentemente atractivo de población migrante, proveniente de las provincias vecinas y parte alta de la provincia de El Oro, lo que produjo el surgimiento de barrios suburbanos en terrenos no urbanizables. Estos terrenos fueron ocupados en forma de "invasión" por familias de escasos recursos económicos con limitado acceso a servicios básicos para el buen vivir. En el contexto del desarrollo nacional, Machala surgió como una de las ciudades intermedias de mayor crecimiento urbano producto de migraciones campo ciudad atraídas por el "boom" bananero (1950), y la actividad camaronera (1970), cuyo crecimiento económico productivo, como es natural, trajo consigo el crecimiento poblacional (Mendoza, 2017).

Lo antes mencionado origina la creación de la parroquia urbana La Providencia considerada una de las más grandes del cantón Machala y la conforman barrios urbanos y suburbanos del sector Norte, Sur, Este de la ciudad. (Barriga, 2010). A pesar del desarrollo económico y productivo de la ciudad de Machala aún no se ha logrado reducir el déficit de servicios básicos elementales para el buen vivir de la población asentada en los barrios marginales de esta parroquia. (Mendoza, 2017)

Lo anotado anteriormente evidencia la segregación territorial, económica y social de los sectores urbano-marginales en la ciudad de Machala que demandan de políticas sociales que permitan integrar al estado de bienestar a la población, y un verdadero desarrollo local sostenible en procura de lograr el Buen vivir. En ese sentido el GAD Cantonal de Machala en su planificación actualizada al 2018 cuenta con una variedad de políticas territoriales enfocadas para las diferentes necesidades locales, consecuentemente citaremos las que tienen que ver con infraestructura y servicios, tales como:

- Regular los asentamientos humanos con características y funciones urbanas, para garantizar el acceso a los servicios e infraestructura básicos.

- Regular y controlar las descargas para asegurar la recuperación integral del entorno.

${ }^{5}$ Ciudades con enormes asimetrías de poder económico frente al resto de ciudades 
- Regular los asentamientos humanos con características y funciones urbanas y rurales, para garantizar el acceso a los servicios e infraestructura básicos.

Cabe mencionar que estas políticas se aplican mediante programas y proyectos a través de los cuales se atiende las necesidades locales del Cantón Machala y están articuladas con los objetivos, políticas y metas del Plan Nacional de Desarrollo (PND), con los objetivos estratégicos, con las categorías de ordenamiento territorial, con la visión, escenarios y políticas locales y especialmente con la participación social como potencialidades de los componentes del Sistema Territorial.

Sin embargo, estas políticas no están siendo aplicadas en los barrios marginales, pese a constar en el Plan de Desarrollo y Ordenamiento territorial del GAD Machala, como se demuestra más adelante en los resultados de la investigación empírica.

\section{Metodología}

Para el desarrollo del estudio se utilizó el tipo de investigación Descriptiva la misma que permitió caracterizar las relaciones de causalidad y plantear un diagnóstico de la realidad de estos sectores segregados. Por su parte el método empírico sirvió para el análisis estadístico que basado en la lógica empírica interpreta la información de campo recopilada mediante instrumentos para indagar los fenómenos sociales, analizarlos, sintetizarlos y generalizar los hallazgos del contexto estudiado.

En lo relacionado al universo objeto de estudio, este lo constituyen 22 barrios urbano marginales de la parroquia La Providencia del Cantón Machala cuyos dirigentes fueron objeto de investigación con los instrumentos estructurados para el efecto, ya que por motivo de emergencia sanitaria (COVID- 19) no se pudo llegar a la población en general, así mismo se aplicó la entrevista a informantes calificados del GAD Municipal del cantón Machala (5 jefes departamentales)

Las técnicas de investigación que se aplicaron son el cuestionario, entrevista y la observación, mismas que se aplicaron mediante instrumentos estructurados (guía de entrevista, boleta de encuesta y guía de observación), para cada técnica, debiendo indicar que la entrevista se aplicó a informantes calificados conocedores de la temática, la observación permitió identificar la problemática in situ y constatar en el entorno todo aquello que no se pudo realizar mediante las otras técnicas.

\section{Resultados y Discusión}

\section{Características de las viviendas que predominan en el sector estudiado.}

Según Pérez (2000) la vivienda surgió en forma simultánea con la sociedad como organización humana, y con la ciudad como manifestación espacial de esta última, está presente en la cotidianidad de la vida de las personas y en la actualidad tener una vivienda 
digna es uno de los derechos humanos fundamentales, toda vez que, según estudios de la ex CONADE en su plan Nacional de desarrollo sostiene que, "el hombre permanece entre el 75 y $80 \%$ del total de su vida en la vivienda y sus inmediaciones, por eso este ambiente merece la atención especial del planificador".

Consecuente con esta información fue interés del estudio indagar sobre las características de las viviendas en los sectores, cuya información se describe en el siguiente gráfico:

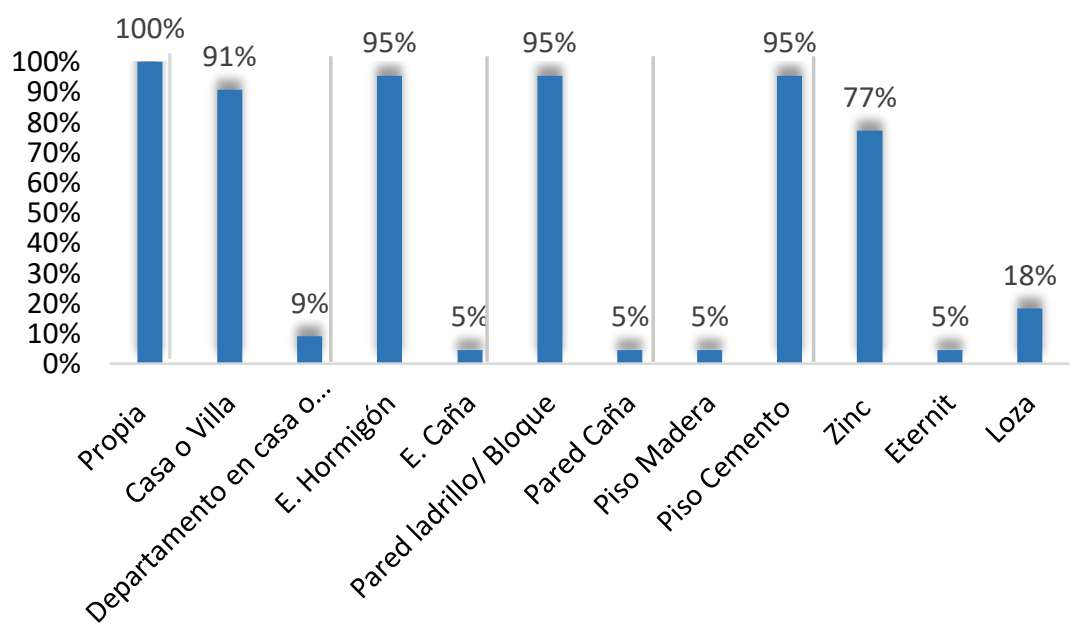

Gráfico 1: Características de las viviendas

Fuente: Investigación directa por la autora

En cuanto a la tenencia se encontró que el 100\% de la población habita en viviendas propias, donde el tipo de vivienda predominante es casa o villa con un 91\%; en cuanto a la estructura de la vivienda el $95 \%$ es de hormigón, consecuentemente comparten importancia porcentual sus paredes de ladrillo o bloque y piso de cemento; en lo relacionado a la cubierta el $77 \%$ es de zinc; el $18 \%$ y $5 \%$ de loza y eternit respectivamente.

Las características antes descritas evidencian que las viviendas reúnen las condiciones de comodidad y seguridad para las familias que la habitan, considerando que estas se constituyen en el lugar donde el ser humano recupera sus energías luego de las jornadas de trabajo en las diferentes actividades productivas.

\section{Servicios básicos e infraestructura comunitaria}

Los servicios básicos representan los componentes esenciales para el desarrollo del ser humano en lo relacionado a la satisfacción de las necesidades que garanticen mejorar la calidad de vida de las personas, actualmente se los reconoce como un derecho humano (UNICEF, 2000). En ese sentido la constitución de la República del Ecuador en el Art. 66, 
numeral 25 menciona que, se reconoce y garantiza a las personas el derecho a acceder a bienes y servicios públicos y privados de calidad, con eficiencia, eficacia y buen trato.

Así mismo el Código Orgánico de Organización Territorial, Autonomía y Descentralización, COOTAD en el Art. 55, literal d) y g) dispone como competencias exclusivas del gobierno autónomo descentralizado municipal, prestar los servicios públicos de agua potable, alcantarillado, depuración de aguas residuales, manejo de desechos sólidos, actividades de saneamiento ambiental. Así como también, planificar, construir y mantener la infraestructura física y los equipamientos de los espacios públicos destinados al desarrollo social, cultural y deportivo, de acuerdo con la ley.

Esto último dispone contar con espacios para prácticas deportivas que garanticen el esparcimiento y confort, así como también lugares de reunión para prácticas religiosas, sociales y culturales de la comunidad. En ese sentido el estudio realizado proporciona los siguientes resultados:

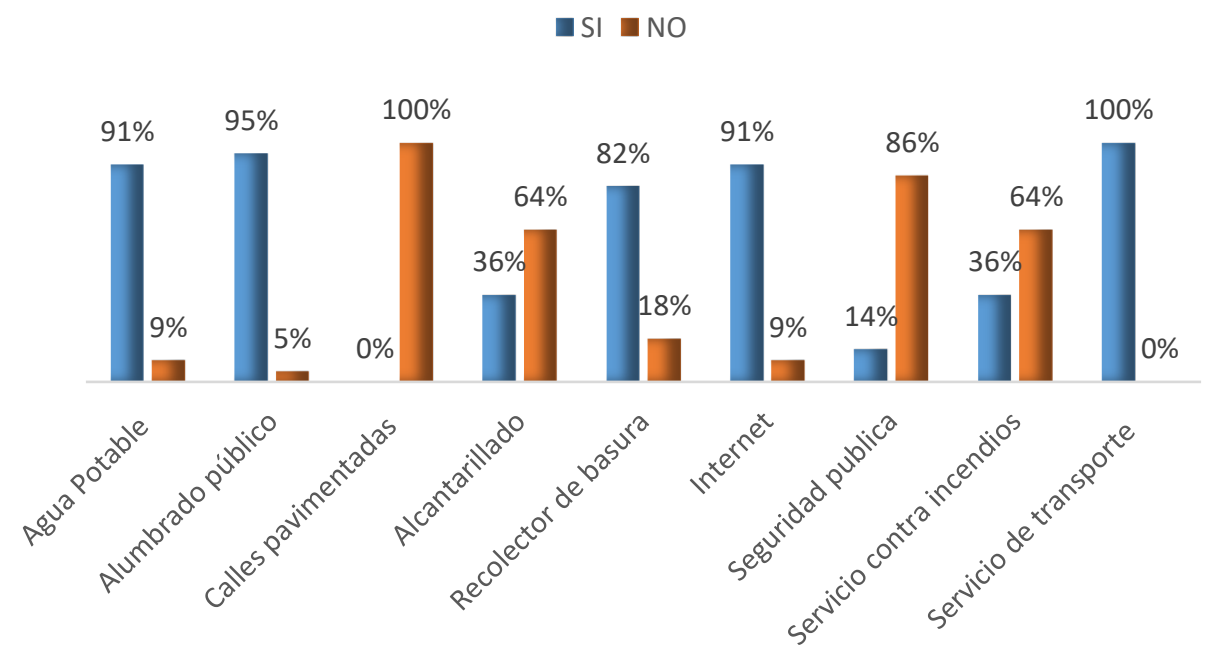

Gráfico 2: Infraestructura física y social

Fuente: Investigación directa por la autora

De

acuerdo a la información proporcionada por moradores y directivos de los sectores investigados en relación a los servicios básicos el 91\% dispone de servicio de agua potable; el alumbrado público dispone un $95 \%$, en cuanto a las calles pavimentadas el $100 \%$ no dispone de esta infraestructura urbanística; así mismo el alcantarillado sanitario solo el $36 \%$ de los sectores cuentan con este servicio, mientras que el servicio de recolección de basura tiene una cobertura del $82 \%$, en lo relacionado a la conectividad, el $91 \%$ dela población cuenta con internet, el servicio de transporte tiene una cobertura de $82 \%$, los servicios de seguridad pública y servicios contra incendios tienen una cobertura de $14 \%$ y $36 \%$ respectivamente. 


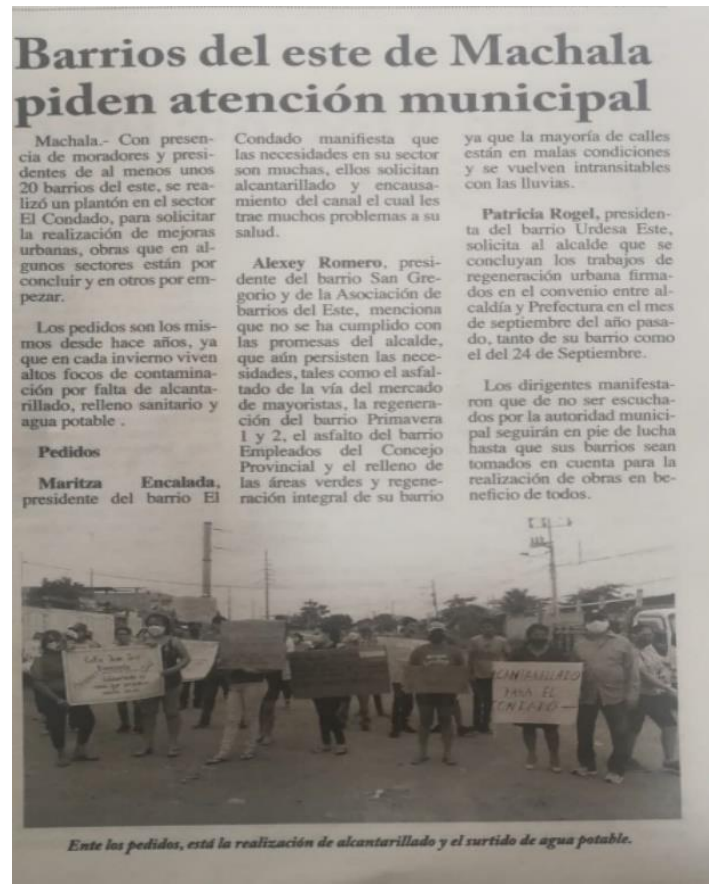

Moradores de sectores urbano marginales de la parroquia La Providencia reclamando servicios básicos.

Diario Opinión

Fecha:13/07/2020

Las estadísticas antes descritas evidencian la carencia de algunos servicios necesarios para mejorar las condiciones de vida, tales como, alcantarillado, calles pavimentadas, seguridad pública y contra incendios, además mediante la observación directa se pudo constatar que gran parte de los sectores no cuentan con infraestructura comunitaria como: casas comunales, centros de educación, estación de autobús, estación de policía, iglesia, mercado, así mismo se evidencia la inexistencia de áreas de esparcimiento adecuado, y como contraste se observa fuentes de insalubridad como solares vacíos llenos de basura, canales de aguas servidas, calles llenas de montes e inseguridad ciudadana.

Sin embargo, al ser consultados los funcionarios municipales responsables de los departamentos indican que existe una planificación en base a los lineamentos generales de los planes nacionales donde se prioriza obras que tienen que ver con el abastecimiento de Infraestructura y servicios básicos, contrastando drásticamente con la realidad que argumentan quienes viven en estos sectores segregados donde dichos servicios no llegan.

Lo anotado anteriormente, anima a sugerir que el GAD del Cantón Machala debe incorporar alguna estrategia para atender este grave problema como es la carencia de servicios básicos indispensables para alcanzar la igualdad que supone el desarrollo sostenible y aplique las políticas públicas que están diseñadas en su planificación.

\section{Acceso a la educación y salud}

Los servicios de educación y salud son fundamentales para el desarrollo de los pueblos, para el caso de Ecuador están garantizados como derechos constitucionales gratuitos, en ese sentido son atendidos por el Gobiernos Nacional, esto ha permitido que los sectores barriales objeto de estudio, tengan acceso un $100 \%$ a estos servicios, como se describe en el siguiente gráfico. 


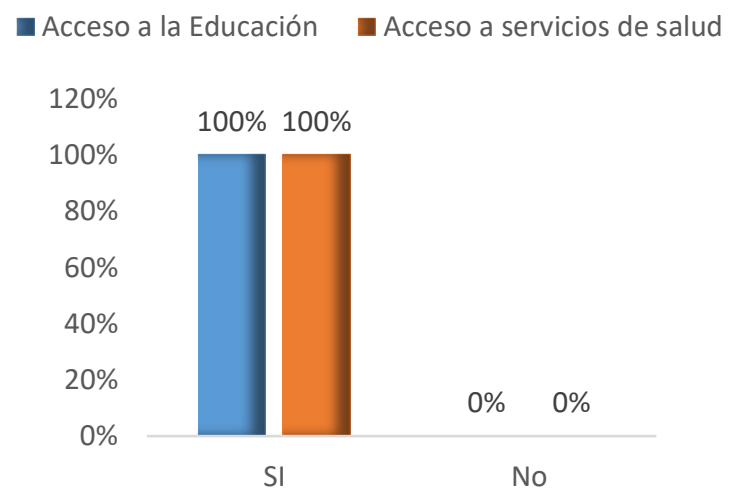

Gráfico 3: Educación y servicios de salud

Fuente: Investigación directa por la autora

\section{Información Económica}

\section{Trabajo adecuado}

La Constitución de la República del Ecuador en su Art. 35. Establece que: El trabajo es un derecho y un deber social y gozará de la protección del Estado, el que asegurará al trabajador el respeto a su dignidad, una existencia decorosa y una remuneración justa que cubra sus necesidades y las de su familia.

Al respecto el Instituto nacional de estadísticas y censos (INEC) en la Encuesta Nacional de Empleo, Desempleo y Subempleo, Indicadores Laborales, define como trabajo adecuado a personas con empleo que, durante la semana de referencia, perciben ingresos laborales iguales o superiores al salario mínimo, trabajan igual o más de 40 horas a la semana, independientemente del deseo y disponibilidad de trabajar horas adicionales.

En ese sentido, el mismo INEC en sus estadísticas laborales ubica a la tasa de empleo adecuado a diciembre 2019 con un $38.8 \%$ y a septiembre 2020 en un $32.1 \%$, lo que implica una disminución de empleos adecuados en 6.7 puntos porcentuales, por su parte el subempleo para el mismo periodo de análisis aumento del $17.8 \%$ a $23.4 \%$ y el desempleo de $3,8 \%$ al $6.6 \%$.

Esta realidad no es ajena a la ciudad de Machala y particularmente a su parroquia La Providencia donde el trabajo adecuado representa el 40\%, lo que implica que el $55 \%$ de la población de este sector estaría en condiciones de subempleo y un 5\% ha perdido su empleo.

\section{Oportunidades laborales de los habitantes de barrios marginales}

En relación a las oportunidades laborales de las personas que habitan en los sectores de estudio manifiestan que vivir en un barrio urbano marginal no limita acceder a un empleo, ya que son diferentes las actividades económicas en las que se desenvuelven cada uno de sus 
moradores, sin embargo, la situación económica actual del país impide acceder a un trabajo adecuado y como se señaló anteriormente el subempleo y el desempleo va en aumento.

\section{Ocupación del jefe del hogar y rango aproximado de ingresos}

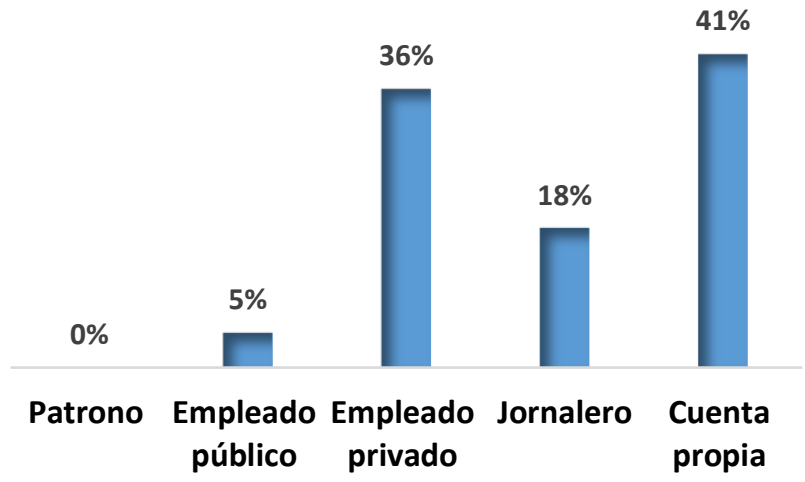

Gráfico 4: Ocupación del jefe del hogar

Fuente: Investigación directa por la autora

La categoría ocupacional de los jefes de hogar de los sectores de estudio se ubica el trabajador cuenta propia con un $45 \%$, seguido del empleado privado con $32 \%$, el jornalero $18 \%$ y en un porcentaje inferior el empleado público con un $5 \%$.

La información estadística del sector ratifica lo anunciado anteriormente, esto es, el aumento en los índices de subempleo que traducido significa ese gran porcentaje de trabajadores por cuenta propia que han desarrollado estrategias de subsistencia laboral.

\section{Ingreso mensual}

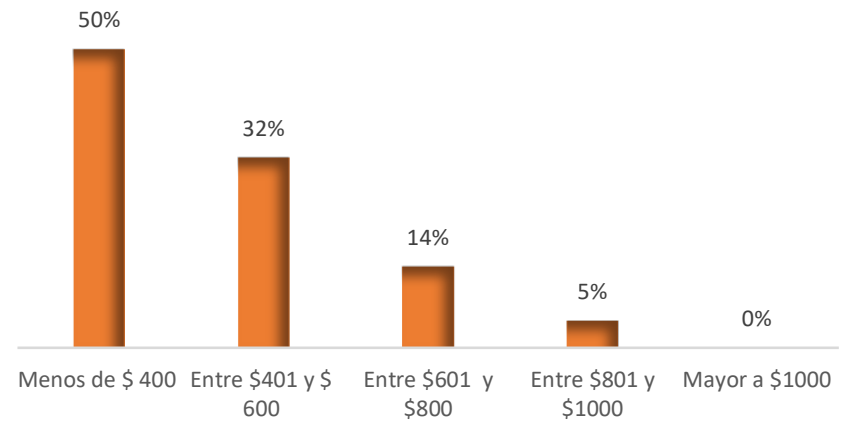

Gráfico 5: Ingreso mensual

Fuente: Investigación directa por la autora 
De la población Económicamente activa (PEA) ocupada del sector de estudio, esta registra un ingreso mensual inferior al salario mínimo vital $(\$ 400.00)$ y representan el $50 \%$ de encuestados, por otra parte, existe $32 \%$ con ingresos que fluctúan entre $\$ 400$ y $\$ 600$, seguido de un $14 \%$ de ingresos que están entre $\$ 601$ y $\$ 800$ y un 5\% entre $\$ 801$ y $\$ 1000$. Esto significa que, como anteriormente se expuso, habido disminución en los índices de empleo adecuado lo que ha generado incremento de subempleo consecuentemente bajos salarios que no cubren la canasta básica familiar, en el caso que nos ocupa estamos frente a un $82 \%$ de personas cuyos ingresos no cubren la misma.

\section{Apreciación de los moradores de las condiciones comunitarias del área de estudio en relación con otros barrios de la ciudad}

Los moradores de los sectores urbano marginales de la parroquia La Providencia, manifiestan que otros barrios si se encuentran en mejores condiciones, ya que estos han sido abastecidos de servicios básicos e infraestructura comunitaria como, alcantarillado, agua, energía eléctrica, bordillos y asfalto. A más de ello cuentan con espacios recreativos como canchas, parques, también existen casas comunales, estación de policía, cámaras de vigilancia, esta situación ha generado desigualdad y fragmentación social entre barrios, por un lado los que han sido beneficiados por el clientelismo político y por otro lado los marginales que no tuvieron una representación o participación política con los gobernantes de los Gobiernos Locales cuyos servicios básico e infraestructura siguen postergados, pese a que estos sectores tiene entre 10 y 38 años de creación.

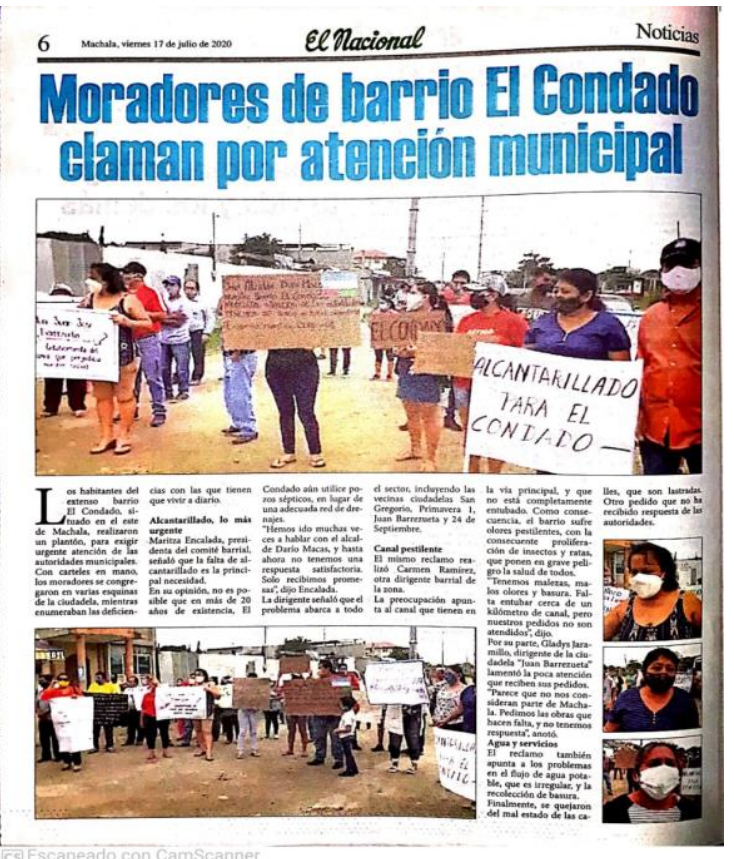

Moradores de uno de los barrios de la Parroquia la Providencia reclamando atención a sus necesidades

Diario

Nacional

Fecha: $16 / 07 / 2020$

Sin embargo, la Municipalidad afirma que de manera plurianual se realizan asambleas en la que en base a las necesidades que cada dirigente barrial expone sobre su sector se elabora un presupuesto, información que de acuerdo a los moradores de los sectores estudiados ciertamente se realiza esta asamblea, pero como mero justificativo del Gobierno Local porque por mandado de ley se debe elaborar de esa manera el presupuesto, pero las obras no llegan. 


\section{Niveles de escolaridad de los habitantes del área de estudio}

La Ley Orgánica de educación intercultural (LOEI), establece que el sistema nacional de educación, comprende los niveles de educación inicial, básica y bachillerato; el sistema de educación superior y el sistema de educación intercultural bilingüe. Y en el Art. 7, literal b) señala que es un derecho de la y los estudiantes recibir una formación integral y científica, que contribuya al pleno desarrollo de su personalidad, capacidades y potencialidades, respetando sus derechos, libertades fundamentales y promoviendo la igualdad de género, la no discriminación, la valoración de las diversidades, la participación, autonomía y cooperación.

En tal virtud y consecuente con estos postulados la población de los sectores objeto de estudio evidencian los niveles antes descritos en el siguiente gráfico.

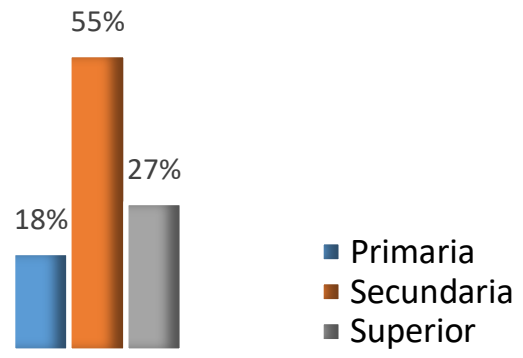

Gráfico 6: Nivel de Instrucción

Fuente: Investigación directa por la autora

La información estadística evidencia que la población objeto de estudio tiene un nivel de educación primaria del $18 \%$, secundaria con $55 \%$ y superior con un $27 \%$, esto implica que el grado de escolaridad es aceptable debido a las facilidades que la modernidad brinda para el acceso a la educación, a más de las exigencias que esta misma implica para lograr insertarse al mercado laboral.

\section{Auto identificación étnica}

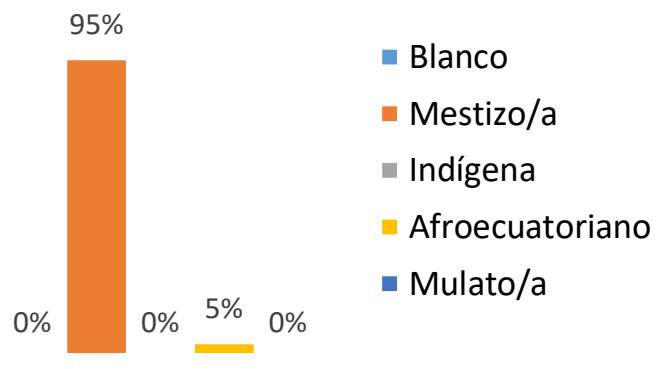

Fuente: Investigación directa por la autora

Gráfico 7: Autoidentificación 
En el Ecuador la mayoría de la su población se auto identifica étnicamente como mestiza, y la población de la ciudad de Machala no es ajena a esta realidad, para el caso de la Parroquia urbana la Providencia el 95\% de la población es mestiza y un 5\% se auto identifica como Afro Ecuatoriano.

\section{Propuesta}

Retomando lo citado anteriormente, esto es, la Constitución de la República del Ecuador que establece, el desarrollo estará orientado a la realización del Buen Vivir que promueve una visión basada en la dignidad humana, en el libre ejercicio y disfrute de los derechos, bajo esta visión, convierte al ser humano como el centro y fin último, esta perspectiva demanda de políticas integrales tendientes a lograr condiciones de igualdad. En ese sentido el instrumento máximo de la planificación nacional, Plan Nacional de Desarrollo (PND) contiene lineamientos y políticas formuladas en consonancia con un enfoque basado en derechos humanos, cuyos principios están orientados al Buen Vivir y goce de los derechos de las y los ecuatorianos, conforme establece el artículo 85 de la Constitución.

Por su parte el GAD Cantonal en su planificación cuenta con políticas enfocadas a mejorar la calidad de vida de sus habitantes, sin embargo, en su aplicación surgen dificultades que limitan su ejecución, entre las que se destacan: el clientelismo político, nula decisión política para los sectores marginales en la dotación de servicios básicos, escaza priorización de obras vitales para el desarrollo humano, distribución inequitativa de los recursos entre sectores poblacionales, sumado a esto, la limitada planificación en función de los sectores más necesitados y los recortes presupuestarios por parte del Gobierno Nacional a los Gobiernos Locales.

En tal virtud es imperativo formular una política pública encaminada a menguar las dificultades antes descritas, en ese sentido se propone "Incorporar procesos de seguimiento y participación ciudadana en la aplicación de estas políticas" de esta manera ejercer un control de las falencias que impiden atender a los sectores más necesitados.

Cabe indicar que la propuesta se desprende del diagnóstico del área de estudio donde evidencia que los problemas de servicios básicos fundamentales persisten, pese a que en el PD y OT del cantón Machala existe la Política pública para acometer estas necesidades.

\section{Conclusiones}

- La desigualdad social entre el área objeto de estudio y los sectores vecinos, no solo implica la concentración de pobreza si no que, además, ha conllevado una diferencia objetiva entre ellos, lo que se refleja en la desigualdad de equipamientos comunitarios y servicios de calidad, toda vez que existen barrios que tienen entre 10 y 38 años de creación y sus necesidades no han sido atendidas, lo que evidencia segregación 
urbana visto que es evidente las disparidades sociales y geográficas en estos sectores, lo que limita lograr el desarrollo local sostenible e integración social.

- La carencia de algunos servicios necesarios para mejorar las condiciones de vida es evidente en el sector, tales como, falta de alcantarillado, calles pavimentadas, seguridad pública y contra incendios, en relación a la infraestructura comunitaria carecen de casas comunales, centros de educación, estación de autobús, estación de policía, iglesia, mercado, de igual forma se evidencia la inexistencia de áreas de esparcimiento adecuado, y como contraste se observa fuentes de insalubridad como solares vacíos llenos de basura, canales de aguas servidas, calles llenas de montes e inseguridad ciudadana.

- La planificación del GAD los funcionarios sostienen que se realiza en base a los lineamentos generales de los planes nacionales donde se prioriza obras que tienen que ver con el abastecimiento de Infraestructura y servicios básicos, sin embargo, está muy distante de la realidad que viven estos sectores segregados donde los servicios básicos no llegan.

- Frente a los problemas de mayor impacto en la comunidad se sugiriere aplicar las políticas territoriales establecidas en el Plan de Desarrollo y Ordenamiento Territorial del GAD Machala relacionadas con la atención a servicios básicos e infraestructura comunitaria, que deberían ser implementadas por el Gobierno Local para solucionar estas necesidades insatisfechas.

\section{Referencias Bibliográficas}

Abad, G. (2013). Medios, periodismo y responsabilidad social. Chasqui: Revista Latinoamericana de Comunicación, ISSN 1390-1079, ISSN-e 1390-924X, No. 122, 2013 (Ejemplar Dedicado a: Medios, Periodismo y Responsabilidad Social), Págs. 13-22, 122, 13-22. https://dialnet.unirioja.es/servlet/articulo?codigo=5791074

Aguado Moralejo, I., Echebarria Miguel, C., \& Barrutia Legarreta, J. (2009). Recorrido Teórias Económicas. Revista de Economía Mundial, 21, 87-110. https://doi.org/86611886004

Albornoz, V. (2011). Crecimiento y Progreso Social en Chile. In Temas de Economía y Política (Primera, Vol. 202, Issue 562). https://www.losexplicadores.com/vicente/articulos/15_Crecimiento_y_progreso_socia 1.pdf

Alburquerque, F. (2004). El Enfoque del Desarrollo Económico Local. In Desarrollo Económico Local y Empleabilidad (Primera ed). Organización Internacional del Trabajo 2004.

https://www.flacsoandes.edu.ec/sites/default/files/agora/files/1251776298.area_enfoqu e_del_0.pdf 
Arocena, J. (2002). El desarrollo local : un desafio contemporáneo (1995 Centro Latinoamericano de Economía Humana (ed.); ilustrada).

Asamblea General de las Naciones Unidas. (2012). Desarrollo sostenible. https://www.un.org/es/ga/president/65/issues/sustdev.shtml

Barriga Gómez, D. (2010). Estudio Investigativo Sobre La Cultura Gastronómica De La Ciudad De Machala, Sus Costumbres Y Formas De Alimentación [Universidad Tecnológica Equinoccial].

http://repositorio.ute.edu.ec/xmlui/bitstream/handle/123456789/11576/42632_1.pdf?se quence $=1 \&$ is Allowed $=\mathrm{y}$

Boada Ortiz, A., Rocchi, S., \& Kuhndt, M. (2005). Negocios Y Sostenibilidad Más Allá De La Gestión Ambiental (@Fundación Politécnico Grancolombiano 2005 (ed.); Primera Ed). http://alejandria.poligran.edu.co/bitstream/handle/10823/781/Negocios y sostenibilidad.pdf?sequence $=1 \&$ isAllowed $=y$

Carpio, J., Carrión, D., Jácome, N., García, J., Carrión, F., Pérez, J. ., Rodriguez, A., Villavicencio, G., \& Menéndez, A. (1987). El proceso urbano en el Ecuador. El Proceso Urbano En El Ecuador, 123-150.

Europea, U. (2008). Libro Verde sobre la cohesión territorial. 1-16. https://ec.europa.eu/regional_policy/archive/consultation/terco/pdf/4_organisation/90_ odta_es.pdf

Gallicchio, E. (2010). El Desarrollo Local: ¿Territorializar Políticas O Genera Políticas Territoriales?. Reflexiones Desde La Práctica. Eutopía - Revista de Desarrollo Económico Territorial, 1, 11-23. https://doi.org/10.17141/eutopia.1.2010.933

García Batiz, M., Flores Payán, L., \& Venegas Sahagún, B. (2016). Análisis del desarrollo sostenible en espacios locales. Aplicación de la teoría de conjuntos difusos. Íconos: Revista de Ciencias Sociales, 54, 171-195.

Godoy, A. (2019). Integración social: ¿oportunidad de que familias de escasos recursos vivan en sectores de mayores ingresos y equipamientos? una mirada a las posibilidades que entregan el mercado, el estado y la vía de la informalidad. Eure, 45(136), 71-92. https://doi.org/10.4067/S0250-71612019000300071

González-Lendizabal, K., Farfan-Escalera, R., \& Perez-RamÍrez, \& C. (2016). Elementos del desarrollo local y recursos disponibles para el desarrollo del turismo alternativo en Ocuilan, México. 12, 20.

Irausquín, C., Colina, J., Moreno, D., \& Marín, F. (2017). Fundamentos conceptuales del desarrollo. Multiciencias, 16(3), 288-293. 
https://www.redalyc.org/pdf/904/90453464007.pdf

Jiménez, M. Á. C., \& Espejel, B. O. (2019). Políticas públicas de desarrollo regional para el cambio climático hacia el 2020 en contextos de marginación y de migración internacional. Estudios Sociales. Revista de Alimentación Contemporánea y Desarrollo Regional, 29, 29. https://doi.org/http://dx.doi.org/10.24836/es.v29i53.703

Larraín, C., \& Razmilic, S. (2019). Notas sobre proyecto de ley de integración social y urbana. 20. www.cepchile.cl

Madrid, C. P. M., Ramoneda, Á., \& Valenzuela, F. (2019). La Integración Social Como Desafío: Análisis Del Programa De Campamentos En Chile (2011-2018). Revista INVI, 34(97), 49-78. https://doi.org/10.4067/S0718-83582019000300049

Mendieta Muñoz, R. (2009). Lectura para una revisión de los estilos de desarrollo económico implementados en américa latina y el ecuador. 1-15.

Mendoza, G. (2017). Machala: Realidades urbanas de una ciudad en desarrollo. In Editorial Academica española (Issue Vivencias, problemas y necesidades de carácter económico y social que tiene la población de una ciudad que progresa).

Mora Mayoral, M. J., \& Martínez Martínez, F. R. (2018). Desarrollo local sostenible, responsabilidad social corporativa y emprendimiento social. Equidad y Desarrollo, 31, 27-46. https://doi.org/10.19052/ed.4375

Morales Pérez, M. (2006). El desarrollo local sostenible. Economía y Desarrollo, 140, 13.

Mujica, N., \& Rincón, S. (2010). El concepto de desarrollo: posiciones teóricas más relevantes. 17. http://ve.scielo.org/scielo.php?script=sci_arttext\&pid=S131599842010000200007

Pérez, A. M. R. (2000). Aspectos Teóricos De La Vivienda En Relación Al Habitar. Revista INVI, 15(0718-8358), 20. http://revistainvi.uchile.cl/index.php/INVI/article/view/263/782

Rodrigo, P., \& Arenas, D. (2014). La nueva gobernanza política y las colaboraciones intersectoriales para el desarrollo sostenible. Innovar, 24(53), 197-210. https://doi.org/10.15446/innovar.v24n53.43945

Rodríguez, J., \& Arriagada, C. (2004). Segregación residencial en la ciudad latinoamericana. In EURE (Santiago): Vol. XXIX (Issue c). https://doi.org/10.4067/S0250-71612004008900001

Sánchez Steiner, L. M. (2008). Éxodos rurales y urbanización en Colombia. Perspectiva Histórica Y Aproximaciones Teóricas. Revista Bitácora Urbano Territorial, 13(2), 
$57-72$.

Secretaria Tecnica Planifica Ecuador, S. (2019). Informe de avance del cumplimiento de la Agenda 2030 para el Desarrollo Sostenible 2019.

https://www.planificacion.gob.ec/wp-content/uploads/downloads/2019/07/InformeAvance-Agenda-2030-Ecuador-2019.pdf

Sen, A. K. (1998). Las teorías del desarrollo a principios del siglo XXI. Revista Cuadernos De Economía, 29, 73-200. http://econpapers.repec.org/RePEc:col:000093:007577

UNICEF. (2000). ¿Servicios básicos para todos? El gasto público y la dimensión social de la pobreza. In Servicios básicos para todos? El gasto público y la dimensión social de la pobreza.

Velásquez Gavilanes, R. (2009). Hacia una nueva definición del concepto "política pública." Desafíos, 20, 149-187.

https://www.redalyc.org/pdf/3596/359633165006.pdf

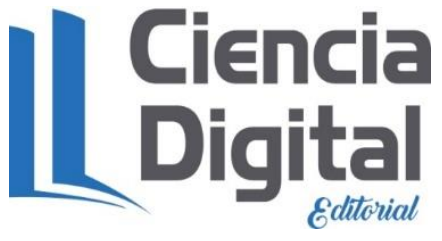


PARA CITAR EL ARTÍCULO INDEXADO.

Granda Granda, A. V., Carriel Bustamante, V. V., \& Castillo Ortega, Y. (2021). La integración social: Un desafío en la política de desarrollo local sostenible en la parroquia urbana la providencia del cantón Machala. Ciencia Digital, 5(1), 158-181. https://doi.org/10.33262/cienciadigital.v5i1.1528

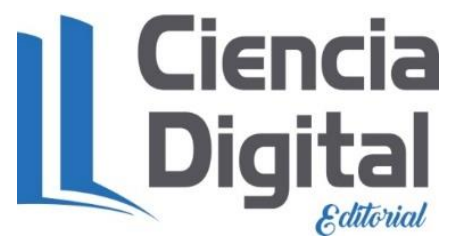

El artículo que se publica es de exclusiva responsabilidad de los autores y no necesariamente reflejan el pensamiento de la Revista Ciencia Digital.

El artículo queda en propiedad de la revista y, por tanto, su publicación parcial y/o total en otro medio tiene que ser autorizado por el director de la Revista Ciencia Digital.
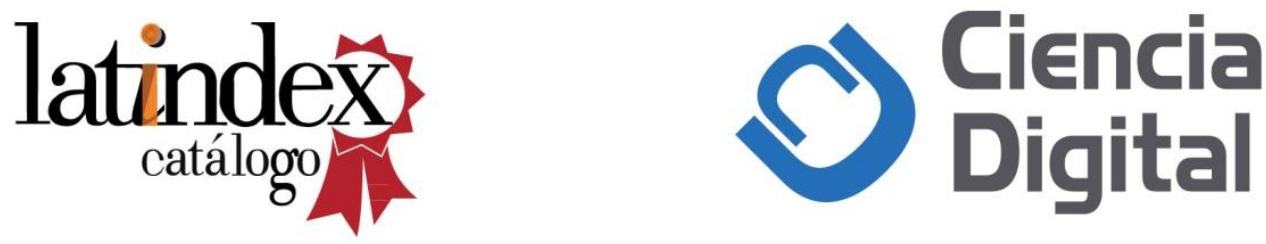